\title{
Two Node Tandem Queueing Model with Phase Type State and Time Dependent Service Rates
}

\author{
K. Srinivasa Rao \\ Department of Statistics \\ Andhra University, Visakhapatnam, \\ India
}

\author{
J. Durga Aparajitha \\ Department of Statistics, \\ Andhra University, Visakhapatnam, \\ India
}

\begin{abstract}
This paper develops and analyzes a two node tandem queueing model with phase type service having time and state dependent service rates. Here, it is assumed that the service processes of the two service stations follow non-homogenous Poisson processes and service rates are dependent on the number of customers in the queue connected to it. Using the difference-differential equations, the joint probability generating function of the queue size distribution is derived. The system performance measures such as average number of customers in the queue, throughput of the service stations, and average waiting time of customers in the queue and in the system and the variance of the number of customers in each queue are derived. A numerical illustration is presented. The sensitivity analysis of the model revealed that the time and load dependent service rates have significant influence on congestion of queues and waiting time. The transient analysis can predict the performance measures more accurately for small period of time. This model can also include some of the early models as particular cases.
\end{abstract}

\section{Keywords}

Time dependent service rate, tandem queueing model, Nonhomogeneous Poisson processes, performance measures, sensitivity analysis

\section{INTRODUCTION}

Queueing models create lot of interest due to their ready applicability in several areas such as communication networks, transportation systems, cargo handling and machine repair. In queueing models, it is customary to assume that arrival and service processes are independent of time. But in many practical situations arising at place like telecommunications the arrival and service rates are dependent on time. Identifying this gap much work has been reported in literature regarding queueing models with time varying rates. Newell (1968) has studied time-dependent arrival rates. Rothkopf and Oren (1979) have given closure approximation for the non-stationary M/M/s queue. Massey et al. (1993) have studied networks of infinite- server queues with non-stationary Poisson input, queueing systems. Massey and Whitt (1994) have analyzed of the modified offered load approximation for the non-stationary Erlang loss model. Mandelbaum and Massey (1995) have studied the approximation for time dependent queues. Davis et al. have (1995) studied the sensitivity to the service time distribution in the non-stationary Erlang loss model. William A. Massey (1996) has studied stability of queues with time varying rates. He analyzed the model using asymptotic method known as Uniform acceleration asymptotic behavior carry based on fluid and diffusion approximations. Duffield et al. (2001) have analyzed a non-stationary offered load model for packet networks. William A. Massey (2002) has analyzed the queues with time varying rates for telecommunication models. He also reviewed several woks which support the arguments that time dependent behavior has an impact on traffic flow models. Ward Whitt (2016) reviewed the recent papers on time varying single server queue. In all these papers they analyzed the queueing models for time varying arrival and service rates using diffusion approximations or Kendal's frame work. Deviating from this Durga Aparajitha and Raj Kumar (2014) developed single server queueing model with time and state dependent service rates using kolomogrov's forward equation approach. They assumed that the service process follows a non-homogeneous Poisson process and depends on number of customers in the queue.

But, in many practical situations, queues in series or tandem queues are more important than analysis of single queues. The output of one queue becomes the input of other queue in tandem queueing models. For example, in communication networks the queues are connected in tandem. Even though much work has been reported in communication networks, very little work has been reported regarding tandem queueing model with time dependent service rates. (Srinivas Rao et al. (2000), Srinivas Rao et al. (2009), Srinivas Rao et al. (2011), Suhasini et al. (2012), Sadu, A. R. et al. (2017)). In self similarity networks the service rates are made time dependent in order to reduce congestion in queues and burstness of buffer.

Hence, in this paper we develop and analyze a two node tandem queueing model having phase type service with time and state depend service rate. Here, it is assumed that the arrival process follows Poisson process with parameter $\lambda$, and service processes follows non-homogeneous Poisson processes. Using the kolomogrov's forward type equations the joint probability generating function of the queue size distributions is obtained. The characteristics of queueing model such as average number of customers in each queue, the utilization of service station, the throughput of the service station, average waiting time of the customers and the variance of the number of customers in the queue and in the system are obtained. A numerical illustration demonstration the solution procedure of the model and the sensitivity analysis are presented. A comparative study of the model with that of homogeneous service rates is also discussed.

\section{QUEUEING MODEL}

In this section, we briefly present the development of the queueing model under study. Consider a two node tandem queueing model with the following assumptions:

1) The arrival process follows Poisson process with mean arrival rate $\lambda$.

2) The service process follows a non Homogeneous Poisson process with mean service rates $\mu_{1}(\mathrm{t})=\alpha_{1}+\beta_{1} \mathrm{t}$ and $\mu_{2}(\mathrm{t})$ $=\alpha_{2}+\beta_{2}$ t.

3) It is further assumed that the service rate is dependent on the number of customers in the queue.

4) The Queue discipline is fist-in-first-out. 
5) The buffer capacity is infinity.

6) Some customers leave the system after first node some other join the queue connected to the second node.

The schematic diagram representing the queuing model is shown in Fig 1

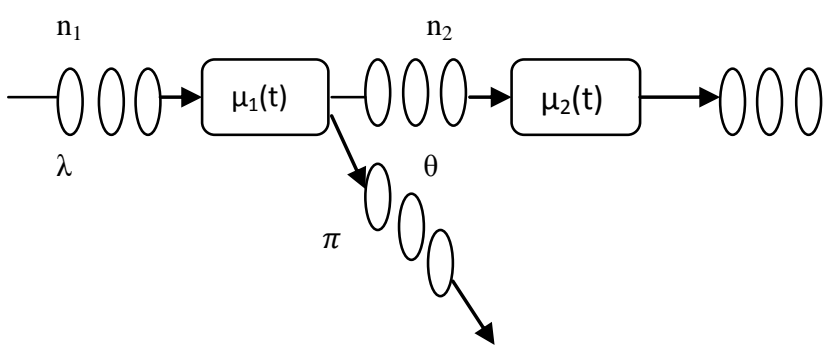

Fig 1 schematic diagram of the queueing model.

Let $\mathrm{n}_{1}$ and $\mathrm{n}_{2}$ denote the number of customers in the first and second queues and $P_{n_{1}, n_{2}}(t)$ be the probability that there are $\mathrm{n}_{1}$ customers in the first queue and $\mathrm{n}_{2}$ customers in the second queue at time $t$.

The difference-differential equations governing the model are:

$$
\begin{aligned}
\frac{\partial P_{n_{1}, n_{2}}(t)}{\partial t}= & -\left(\lambda+n_{1} \mu_{1}(t)+n_{2} \mu_{2}(t)\right) P_{n_{1}, n_{2}}(t) \\
& +\lambda P_{n_{1}-1, n_{2}}(t)+\left(n_{1}+1\right) \mu_{1}(t) \theta P_{n_{1}+1, n_{2}-1}(t) \\
& +\left(n_{1}+1\right) \mu_{1}(t) \pi P_{n_{1}+1, n_{2}}(t) \quad \forall n_{1}, n_{2}>0
\end{aligned}
$$

$$
\begin{aligned}
& \frac{\partial P_{n_{1}, 0}(t)}{\partial t}=-\left(\lambda+n_{1} \mu_{1}(t)\right) P_{n_{1}, 0}(t)+\lambda P_{n_{1}-1,0}(t) \\
&+\left(n_{1}+1\right) \mu_{1}(t) \pi P_{n_{1}+1,0}(t)+\mu_{2}(t) P_{n_{1}, 1}(t) ; \\
& \forall n_{1}>0, n_{2}=0
\end{aligned}
$$

$$
\begin{array}{r}
\frac{\partial P_{0, n_{2}}(t)}{\partial t}=-\left(\lambda+n_{2} \mu_{2}(t)\right) P_{0, n_{2}}(t)+\mu_{1}(t) \theta P_{1, n_{2}-1}(t) \\
+\mu_{1}(t) \pi P_{1, n_{2}}(t)+\left(n_{2}+1\right) \mu_{2}(t) P_{0, n_{2}+1}(t) ; \\
\forall n_{1}=0, n_{2}>0
\end{array}
$$$$
\begin{aligned}
& \frac{\partial P_{0,0}(t)}{\partial t}=-\lambda P_{0,0}(t)+\mu_{1}(t) \pi P_{1,0}(t)+\mu_{2}(t) P_{0,1}(t) ; \\
& \forall n_{1}=0, n_{2}=0
\end{aligned}
$$

probability generating function of $P_{n_{1}, n_{2}}(t)$ be

$$
P\left(S_{1}, S_{2}, t\right)=\sum_{n_{1}=0}^{\infty} \sum_{n_{2}=0}^{\infty} P_{n_{1}, n_{2}}(t) s_{1}{ }^{n_{1}} S_{2}{ }^{n_{2}}
$$

Multiplying the equation (1) with $s_{1}^{n_{1}}, s_{2}^{n_{2}}$ and summing over all $n_{1}, n_{2}$ we get

$$
\begin{aligned}
& \frac{\partial P(t)}{\partial t}=-\sum_{n_{1}=0}^{\infty} \sum_{n_{2}=0}^{\infty}\left(\lambda+n_{1} \mu_{1}(t)+n_{2} \mu_{2}(t)\right) P_{n_{1}, n_{2}}(t) s_{1}{ }^{n_{1}} \\
& s_{2}{ }^{n_{2}}+\sum_{n_{1}=0}^{\infty} \sum_{n_{2}=0}^{\infty} \lambda P_{n_{1}-1, n_{2}}(t) s_{1}{ }^{n_{1}} s_{2}{ }^{n_{2}} \\
& +\sum_{n_{1}=0}^{\infty} \sum_{n_{2}=0}^{\infty}\left(n_{1}+1\right) \mu_{1}(t) \theta P_{n_{1}+1, n_{2}-1}(t) s_{1}{ }^{n_{1}} s_{2}{ }^{n_{2}}
\end{aligned}
$$

$$
\begin{aligned}
& +\sum_{n_{1}=0}^{\infty} \sum_{n_{2}=0}^{\infty}\left(n_{1}+1\right) \mu_{1}(t) \pi P_{n_{1}+1, n_{2}}(t) s_{1}{ }^{n_{1}} s_{2}{ }^{n_{2}} \\
& +\sum_{\substack{n_{1}=0 \\
\text { Substituting } \pi=(1-\theta) \text { simplifying, we get }}}^{\infty}\left(n_{2}+1\right) \mu_{2}(t) P_{n_{1}, n_{2}+1}(t) s_{1}{ }^{n_{1}} s_{2}{ }^{n_{2}} ; \forall n_{1}, n_{2} \\
&
\end{aligned}
$$

$$
\begin{gathered}
\frac{\partial P\left(S_{1}, S_{2}, t\right)}{\partial t}=\mu_{1}(t)\left(1-\theta-s_{1}+\theta s_{2}\right) \frac{\partial P\left(s_{1}, s_{2}, t\right)}{\partial s_{1}} \\
+\mu_{2}(t)\left(1-s_{2}\right) \frac{\partial P\left(s_{1}, s_{2}, t\right)}{\partial s_{2}}-\lambda\left(1-s_{1}\right) P\left(s_{1}, s_{2}, t\right)
\end{gathered}
$$

Solving the equation (4) by Lagrangian's method, the auxiliary equations are:

$$
\begin{gathered}
\frac{d t}{1}=\frac{d s_{1}}{-\mu_{1}(t)\left(1-\theta-s_{1}+\theta s_{2}\right)}=\frac{d s_{2}}{-\mu_{2}(t)\left(1-s_{2}\right)} \\
=\frac{d P}{-\lambda\left(1-s_{1}\right) P\left(s_{1}, s_{2}, t\right)}
\end{gathered}
$$

Considering the service rate is linear and time dependent and they are of the form,

$$
\mu_{1}(t)=\alpha_{1}+\beta_{1}(t) ; 0 \leq \beta_{1} \leq 1
$$

and $\mu_{2}(t)=\alpha_{2}+\beta_{2}(t) ; 0 \leq \beta_{2} \leq 1, \alpha_{1} \neq \alpha_{2}$.

Solving the first and third terms in equation (5), we get

$$
a=\left(s_{2}-1\right) e^{-\int \mu_{2}(t) d t}
$$

Solving the first and second terms in equation (5), we get

$$
\begin{aligned}
b= & s_{1} e^{-\int \mu_{1}(t) d t}+\left(s_{2}-1\right) e^{-\int \mu_{2}(t) d t} \theta \\
& \left(\int \mu_{1}(t) e^{\int\left[\mu_{2}(t)-\mu_{1}(t)\right] d t} d t\right)+\int \mu_{1}(t) e^{-\int \mu_{1}(t) d t} d t
\end{aligned}
$$

Solving the first and fourth terms in the equation (5), we get

$$
\begin{aligned}
c= & P\left(s_{1}, s_{2}, t\right) \exp \left(-\lambda\left(\left[s_{1} e^{-\int \mu_{1}(t) d t}+\left(s_{2}-1\right) e^{-\int \mu_{2}(t) d t}\right.\right.\right. \\
& \left.\theta\left(\int \mu_{1}(t) e^{\int\left[\mu_{2}(t)-\mu_{1}(t)\right] d t} d t\right)+\int \mu_{1}(t) e^{-\int \mu_{1}(t) d t} d t\right] \\
& \int e^{-\int \mu_{1}(t) d t} d t-\left(s_{2}-1\right) e^{-\int \mu_{2}(t) d t} \theta\left(\int e^{\int \mu_{1}(t) d t}\right. \\
& \left.\left(\int \mu_{1}(t) e^{\int\left[\mu_{2}(t)-\mu_{1}(t)\right] d t} d t\right) d t\right)+\int-e^{-\int \mu_{1}(t) d t} \\
& \left.\left.\left(\int \mu_{1}(t) e^{-\int \mu_{1}(t) d t} d t\right) d t-t\right)\right)
\end{aligned}
$$

Where, a, b and c are arbitrary constants. Using the initial conditions

$$
P_{00}(0)=1, P_{00}(t)=0 \forall t>0
$$

The general solution of (5) gives the probability generating function of the number of customers in the first queue and the number of customers in the second queue at time ' $t$ ' is

$$
\begin{gathered}
P\left(s_{1}, s_{2}, t\right)=\exp \left(\lambda \left(( s _ { 1 } - 1 ) e ^ { - ( \alpha _ { 1 } t + \beta _ { 1 } \frac { t ^ { 2 } } { 2 } ) } \left(\int_{0}^{t} e^{\alpha_{1} v+\beta_{1} \frac{v^{2}}{2}} d v\right.\right.\right. \\
\left.-\frac{1}{\alpha_{1}}\right)+\theta\left(s_{2}-1\right) e^{-\left(\alpha_{2} t+\beta_{1} \frac{t^{2}}{2}\right)} \\
\left(\frac{1}{\alpha_{2}-\alpha_{1}}-\frac{\int_{0}^{t}\left(\alpha_{1}+\beta_{1} v\right) e^{\left(\alpha_{2}-\alpha_{1}\right) v+\left(\beta_{2}-\beta_{1} \frac{v^{2}}{2}\right.} d v}{\alpha_{1}}\right)+\theta\left(s_{2}-1\right) \\
e^{-\left(\alpha_{2} t+\beta_{1} \frac{t^{2}}{2}\right)}\left(\int_{0}^{t} e^{\alpha_{1} v+\beta_{1} \frac{v^{2}}{2}} d v \int_{0}^{t}\left(\alpha_{1}+\beta_{1} v\right)\right.
\end{gathered}
$$




$$
\begin{aligned}
& e^{\left(\alpha_{2}-\alpha_{1}\right) v+\left(\beta_{2}-\beta_{1}\right) \frac{v^{2}}{2}} d v-\int_{0}^{t} e^{\alpha_{1} v+\beta_{1} \frac{v^{2}}{2}} \\
& \left.\left.\left.\left(\int_{0}^{t}\left(\alpha_{1}+\beta_{1} v\right) e^{\left(\alpha_{2}-\alpha_{1}\right) v+\left(\beta_{2}-\beta_{1}\right) \frac{v^{2}}{2}} d v\right) d v-\frac{1}{\alpha_{2}}\right)\right)\right)
\end{aligned}
$$

\section{CHARACTERISTIES OF THE QUEUEING MODEL:}

Expanding $P\left(s_{1}, s_{2}, t\right)$ given in equation (6) and collecting the constant terms, we obtain the probability that the queue is empty as

$$
\begin{aligned}
& P_{0,0}(t)= \exp \left(-\lambda \theta\left(e^{-\left(\alpha_{1} t+\beta_{1} \frac{t^{2}}{2}\right)}\right.\right. \\
&\left(\int_{0}^{t} e^{\alpha_{1} v+\beta_{1} \frac{v^{2}}{2}} d v-\frac{1}{\alpha_{1}}\right)+e^{-\left(\alpha_{2} t+\beta_{1} \frac{t^{2}}{2}\right)} \\
&\left(\frac{1}{\alpha_{2}-\alpha_{1}}-\frac{\int_{0}^{t}\left(\alpha_{1}+\beta_{1} v\right) e^{\left(\alpha_{2}-\alpha_{1}\right) v+\left(\beta_{2}-\beta_{1}\right) \frac{v^{2}}{2}} d v}{\alpha_{1}}\right) \\
&+e^{-\left(\alpha_{2} t+\beta_{1} \frac{t^{2}}{2}\right)}\left(\int_{0}^{t} e^{\alpha_{1} v+\beta_{1} \frac{v^{2}}{2}} d v \int_{0}^{t}\left(\alpha_{1}+\beta_{1} v\right)\right. \\
& e^{\left(\alpha_{2}-\alpha_{1}\right) v+\left(\beta_{2}-\beta_{1}\right) \frac{v^{2}}{2}} d v-\int_{0}^{t} e^{\alpha_{1} v+\beta_{1} \frac{v^{2}}{2}}\left(\int_{0}^{t}\left(\alpha_{1}+\beta_{1} v\right)\right. \\
&\left.\left.\left.\left.\left.e^{\left(\alpha_{2}-\alpha_{1}\right) v+\left(\beta_{2}-\beta_{1}\right) \frac{v^{2}}{2}} d v\right) d v\right)-\frac{1}{\alpha_{2}}\right)\right)\right)
\end{aligned}
$$

Taking $\mathrm{s}_{2}=1$ in $P\left(s_{1}, s_{2}, t\right)$, we obtain the probability generating function of the first queue size as

$$
\begin{aligned}
P\left(s_{1}, t\right) & =\exp \left(\lambda\left(s_{1}-1\right) e^{-\left(\alpha_{1} t+\beta_{1} \frac{t^{2}}{2}\right)}\right. \\
& \left(\int_{0}^{t} e^{\alpha_{1} v+\beta_{1} \frac{v^{2}}{2}} d v-\frac{1}{\alpha_{1}}\right) ; \quad \lambda<\alpha_{1}, \beta_{1}
\end{aligned}
$$

By expanding $\mathrm{P}\left(\mathrm{s}_{1}, \mathrm{t}\right)$ and collect the constant terms, we obtain the probability that the first queue is empty as

$P_{0 .}(t)=\exp \left(-\lambda e^{-\left(\alpha_{1} t+\beta_{1} \frac{t^{2}}{2}\right)}\left(\int_{0}^{t} e^{\alpha_{1} v+\beta_{1} \frac{v^{2}}{2}} d v-\frac{1}{\alpha_{1}}\right)\right)$

The mean number of customers in the first system is

$L_{1}(t)=\lambda e^{-\left(\alpha_{1} t+\beta_{1} \frac{t^{2}}{2}\right)}\left(\int_{0}^{t} e^{\alpha_{1} v+\beta_{1} \frac{v^{2}}{2}} d v-\frac{1}{\alpha_{1}}\right)$

The utilization of the first service station is

$U_{1}(t)=1-\exp \left(-\lambda e^{-\left(\alpha_{1} t+\beta_{1} \frac{t^{2}}{2}\right)}\left(\int_{0}^{t} e^{\alpha_{1} v+\beta_{1} \frac{v^{2}}{2}} d v-\frac{1}{\alpha_{1}}\right)\right)$

The throughput of the first service station is

$$
\begin{aligned}
T h P_{1}(t) & =\left(\alpha_{1}+\beta_{1} t\right)(1-\exp \\
& \left.\left(-\lambda e^{-\left(\alpha_{1} t+\beta_{1} \frac{t^{2}}{2}\right)}\left(\int_{0}^{t} e^{\alpha_{1} v+\beta_{1} \frac{v^{2}}{2}} d v-\frac{1}{\alpha_{1}}\right)\right)\right)
\end{aligned}
$$

The average waiting time of a customer in the first system is $W_{1}(t)=\frac{L_{1}(t)}{T \mathrm{~h} P_{1}(t)}$

$$
=\frac{\lambda e^{-\left(\alpha_{1} t+\beta_{1} \frac{t^{2}}{2}\right)}\left(\int_{0}^{t} e^{\alpha_{1} v+\beta_{1} \frac{v^{2}}{2}} d v-\frac{1}{\alpha_{1}}\right)}{\left(\alpha_{1}+\beta_{1} t\right)\left[1-\exp \left(-\lambda e^{-\left(\alpha_{1} t+\beta_{1} \frac{t^{2}}{2}\right)}\left(\int_{0}^{t} e^{\alpha_{1} v+\beta_{1} \frac{v^{2}}{2}} d v-\frac{1}{\alpha_{1}}\right)\right)\right]}
$$

The variance of the number of customer in the first system is

$V_{1}(t)=\lambda e^{-\left(\alpha_{1} t+\beta_{1} \frac{t^{2}}{2}\right)}\left(\int_{0}^{t} e^{\alpha_{1} v+\beta_{1} \frac{v^{2}}{2}} d v-\frac{1}{\alpha_{1}}\right)$

The coefficient of variation of the number of customers in the first system is

$$
C V_{1}(t)=\left(\lambda e^{-\left(\alpha_{1} t+\beta_{1} \frac{t^{2}}{2}\right)}\left(\int_{0}^{t} e^{\alpha_{1} v+\beta_{1} \frac{v^{2}}{2}} d v-\frac{1}{\alpha_{1}}\right)\right)^{-1 / 2}
$$

Taking $\mathrm{s}_{1}=1$ in $P\left(s_{1}, s_{2}, t\right)$ we obtain the probability generating function for the second queue size as

$$
\begin{aligned}
& P\left(s_{2}, t\right)= \exp \left(\lambda \theta \left(( s _ { 2 } - 1 ) e ^ { - ( \alpha _ { 2 } t + \beta _ { 1 } \frac { t ^ { 2 } } { 2 } ) } \left(\frac{1}{\alpha_{2}-\alpha_{1}}\right.\right.\right. \\
&\left.-\frac{\int_{0}^{t}\left(\alpha_{1}+\beta_{1} v\right) e^{\left(\alpha_{2}-\alpha_{1}\right) v+\left(\beta_{2}-\beta_{1}\right) \frac{v^{2}}{2}} d v}{\alpha_{1}}\right)+\left(s_{2}-1\right) \\
& e^{-\left(\alpha_{2} t+\beta_{1} \frac{t^{2}}{2}\right)}\left(\int_{0}^{t} e^{\alpha_{1} v+\beta_{1} \frac{v^{2}}{2}} d v \int_{0}^{t}\left(\alpha_{1}+\beta_{1} v\right)\right. \\
& e^{\left(\alpha_{2}-\alpha_{1}\right) v+\left(\beta_{2}-\beta_{1} \frac{v^{2}}{2}\right.} d v-\int_{0}^{t} e^{\alpha_{1} v+\beta_{1} \frac{v^{2}}{2}} \\
&\left.\left.\left.\left(\int_{0}^{t}\left(\alpha_{1}+\beta_{1} v\right) e^{\left(\alpha_{2}-\alpha_{1}\right) v+\left(\beta_{2}-\beta_{1}\right) \frac{v^{2}}{2}} d v\right) d v-\frac{1}{\alpha_{2}}\right)\right)\right) \\
& \lambda<\min \left(\alpha_{1}+\beta_{1} t\right),\left(\alpha_{2}+\beta_{2} t\right)
\end{aligned}
$$

By expanding $P\left(s_{2}, t\right)$ and collect the constant terms, we obtain the probability that the second queue is empty as

$$
\begin{aligned}
P_{.0}(t)= & \exp \left(\lambda \theta \left(e ^ { - ( \alpha _ { 2 } t + \beta _ { 1 } \frac { t ^ { 2 } } { 2 } ) } \left(\frac{1}{\alpha_{2}-\alpha_{1}}\right.\right.\right. \\
& \left.-\frac{\int_{0}^{t}\left(\alpha_{1}+\beta_{1} v\right) e^{\left(\alpha_{2}-\alpha_{1}\right) v+\left(\beta_{2}-\beta_{1}\right) \frac{v^{2}}{2}} d v}{\alpha_{1}}\right) \\
& e^{-\left(\alpha_{2} t+\beta_{1} \frac{t^{2}}{2}\right)}\left(\int_{0}^{t} e^{\alpha_{1} v+\beta_{1} \frac{v^{2}}{2}} d v \int_{0}^{t}\left(\alpha_{1}+\beta_{1} v\right)\right. \\
& e^{\left(\alpha_{2}-\alpha_{1}\right) v+\left(\beta_{2}-\beta_{1}\right) \frac{v^{2}}{2}} d v-\int_{0}^{t} e^{\alpha_{1} v+\beta_{1} \frac{v^{2}}{2}} \\
& \left.\left.\left.\left(\int_{0}^{t}\left(\alpha_{1}+\beta_{1} v\right) e^{\left(\alpha_{2}-\alpha_{1}\right) v+\left(\beta_{2}-\beta_{1}\right) \frac{v^{2}}{2}} d v\right) d v-\frac{1}{\alpha_{2}}\right)\right)\right)
\end{aligned}
$$

The mean number of customers in the second system is

$$
\begin{aligned}
L_{2}(t) & =\lambda \theta e^{-\left(\alpha_{2} t+\beta_{1} \frac{t^{2}}{2}\right)}\left(\frac{1}{\alpha_{2}-\alpha_{1}}\right. \\
& \left.-\frac{\int_{0}^{t}\left(\alpha_{1}+\beta_{1} v\right) e^{\left(\alpha_{2}-\alpha_{1}\right) v+\left(\beta_{2}-\beta_{1}\right) \frac{v^{2}}{2}} d v}{\alpha_{1}}\right) \\
& +\lambda \theta e^{-\left(\alpha_{2} t+\beta_{1} \frac{t^{2}}{2}\right)}\left(\int_{0}^{t} e^{\alpha_{1} v+\beta_{1} \frac{v^{2}}{2}} d v \int_{0}^{t}\left(\alpha_{1}+\beta_{1} v\right)\right.
\end{aligned}
$$




$$
\begin{aligned}
& e^{\left(\alpha_{2}-\alpha_{1}\right) v+\left(\beta_{2}-\beta_{1}\right) \frac{v^{2}}{2}} d v-\int_{0}^{t} e^{\alpha_{1} v+\beta_{1} \frac{v^{2}}{2}} \\
& \left.\left(\int_{0}^{t}\left(\alpha_{1}+\beta_{1} v\right) e^{\left(\alpha_{2}-\alpha_{1}\right) v+\left(\beta_{2}-\beta_{1}\right) \frac{v^{2}}{2}} d v\right) d v-\frac{1}{\alpha_{2}}\right)
\end{aligned}
$$

The utilization of the second service station is

$$
\begin{gathered}
U_{2}(t)= \\
\left.-\frac{\int_{0}^{t}\left(\alpha_{1}+\beta_{1} v\right) e^{\left(\alpha_{2}-\alpha_{1}\right) v+\left(\beta_{2}-\beta_{1}\right) \frac{v^{2}}{2}} d v}{\alpha_{1}}\right) \\
e^{-\left(\alpha_{2} t+\beta_{1} \frac{t^{2}}{2}\right)}\left(\int_{0}^{t} e^{\alpha_{1} v+\beta_{1} \frac{v^{2}}{2}} d v \int_{0}^{t}\left(\alpha_{1}+\beta_{1} v\right)\right. \\
e^{\left(\alpha_{2}-\alpha_{1}\right) v+\left(\beta_{2}-\beta_{1}\right) \frac{v^{2}}{2}} d v-\int_{0}^{t} e^{\alpha_{1} v+\beta_{1} \frac{v^{2}}{2}} \\
\left.\left.\left.\left(\int_{0}^{t}\left(\alpha_{1}+\beta_{1} v\right) e^{\left(\alpha_{2}-\alpha_{1}\right) v+\left(\beta_{2}-\beta_{1}\right) \frac{v^{2}}{2}} d v\right) d v-\frac{1}{\alpha_{2}}\right)\right)\right)
\end{gathered}
$$

The throughput of the second service station is

$$
\begin{aligned}
& T h P_{2}(t)=\left(\alpha_{2}+\beta_{2} t\right) \\
&\left(1-\exp \left(\lambda \theta \left(e ^ { - ( \alpha _ { 2 } t + \beta _ { 1 } \frac { t ^ { 2 } } { 2 } ) } \left(\frac{1}{\alpha_{2}-\alpha_{1}}\right.\right.\right.\right. \\
&-\left.\frac{\left.\int_{0}^{t}\left(\alpha_{1}+\beta_{1} v\right) e^{\left(\alpha_{2}-\alpha_{1}\right) v+\left(\beta_{2}-\beta_{1}\right) \frac{v^{2}}{2}} d v\right)}{\alpha_{1}}\right) \\
& e^{-\left(\alpha_{2} t+\beta_{1} \frac{t^{2}}{2}\right)}\left(\int_{0}^{t} e^{\alpha_{1} v+\beta_{1} \frac{v^{2}}{2}} d v \int_{0}^{t}\left(\alpha_{1}+\beta_{1} v\right)\right. \\
& e^{\left(\alpha_{2}-\alpha_{1}\right) v+\left(\beta_{2}-\beta_{1}\right) \frac{v^{2}}{2}} d v-\int_{0}^{t} e^{\alpha_{1} v+\beta_{1} \frac{v^{2}}{2}} \\
&\left.\left.\left.\left.\left(\int_{0}^{t}\left(\alpha_{1}+\beta_{1} v\right) e^{\left(\alpha_{2}-\alpha_{1}\right) v+\left(\beta_{2}-\beta_{1}\right) \frac{v^{2}}{2}} d v\right) d v-\frac{1}{\alpha_{2}}\right)\right)\right)\right)
\end{aligned}
$$

The average waiting time of a customer in the second system is

$$
\mathrm{W}_{2}(\mathrm{t})=\frac{\mathrm{L}_{2}(\mathrm{t})}{\operatorname{Thp}_{2}(\mathrm{t})}
$$

where, $\mathrm{L}_{2}(\mathrm{t})$ and $\mathrm{Thp}_{2}(\mathrm{t})$ are as given in equations (18) and (20) respectively.

The variance of the number of customer in the second system is

$$
\begin{aligned}
& V_{2}(t)= \lambda \theta e^{-\left(\alpha_{2} t+\beta_{1} \frac{t^{2}}{2}\right)}\left(\frac{1}{\alpha_{2}-\alpha_{1}}\right. \\
&\left.-\frac{\int_{0}^{t}\left(\alpha_{1}+\beta_{1} v\right) e^{\left(\alpha_{2}-\alpha_{1}\right) v+\left(\beta_{2}-\beta_{1}\right) \frac{v^{2}}{2}} d v}{\alpha_{1}}\right) \\
&+\lambda \theta e^{-\left(\alpha_{2} t+\beta_{1} \frac{t^{2}}{2}\right)}\left(\int_{0}^{t} e^{\alpha_{1} v+\beta_{1} \frac{v^{2}}{2}} d v \int_{0}^{t}\left(\alpha_{1}+\beta_{1} v\right)\right. \\
& e^{\left(\alpha_{2}-\alpha_{1}\right) v+\left(\beta_{2}-\beta_{1}\right) \frac{v^{2}}{2}} d v-\int_{0}^{t} e^{\alpha_{1} v+\beta_{1} \frac{v^{2}}{2}} \\
&\left.\left(\int_{0}^{t}\left(\alpha_{1}+\beta_{1} v\right) e^{\left(\alpha_{2}-\alpha_{1}\right) v+\left(\beta_{2}-\beta_{1}\right) \frac{v^{2}}{2}} d v\right) d v-\frac{1}{\alpha_{2}}\right)
\end{aligned}
$$

The coefficient of variation of the number of customers in the second system is

$$
\begin{aligned}
& C V_{2}(t)=\left(\lambda \theta e ^ { - ( \alpha _ { 2 } t + \beta _ { 1 } \frac { t ^ { 2 } } { 2 } ) } \left(\frac{1}{\alpha_{2}-\alpha_{1}}\right.\right. \\
&\left.-\frac{\int_{0}^{t}\left(\alpha_{1}+\beta_{1} v\right) e^{\left(\alpha_{2}-\alpha_{1}\right) v+\left(\beta_{2}-\beta_{1}\right) \frac{v^{2}}{2}} d v}{\alpha_{1}}\right) \\
&+\lambda \theta e^{-\left(\alpha_{2} t+\beta_{1} \frac{t^{2}}{2}\right)}\left(\int_{0}^{t} e^{\alpha_{1} v+\beta_{1} \frac{v^{2}}{2}} d v \int_{0}^{t}\left(\alpha_{1}+\beta_{1} v\right)\right. \\
& e^{\left(\alpha_{2}-\alpha_{1}\right) v+\left(\beta_{2}-\beta_{1} \frac{v^{2}}{2}\right.} d v-\int_{0}^{t} e^{\alpha_{1} v+\beta_{1} \frac{v^{2}}{2}} \\
&\left(\int_{0}^{t}\left(\alpha_{1}+\beta_{1} v\right) e^{\left(\alpha_{2}-\alpha_{1}\right) v+\left(\beta_{2}-\beta_{1}\right) \frac{v^{2}}{2}} d v\right) d v \\
&\left.\left.-\frac{1}{\alpha_{2}}\right)\right)^{-1 / 2} * 100
\end{aligned}
$$

The mean number of customers in the queueing system at time $\mathrm{t}$ is

$$
\begin{aligned}
& L(t)= L_{1}(t)+L_{2}(t) \\
&= \lambda e^{-\left(\alpha_{1} t+\beta_{1} \frac{t^{2}}{2}\right)}\left(\int_{0}^{t} e^{\alpha_{1} v+\beta_{1} \frac{v^{2}}{2}} d v-\frac{1}{\alpha_{1}}\right) \\
& \lambda \theta e^{-\left(\alpha_{2} t+\beta_{1} \frac{t^{2}}{2}\right)}\left(\frac{1}{\alpha_{2}-\alpha_{1}}\right. \\
&\left.-\frac{\int_{0}^{t}\left(\alpha_{1}+\beta_{1} v\right) e^{\left(\alpha_{2}-\alpha_{1}\right) v+\left(\beta_{2}-\beta_{1}\right) \frac{v^{2}}{2}} d v}{\alpha_{1}}\right) \\
&+\lambda \theta e^{-\left(\alpha_{2} t+\beta_{1} \frac{t^{2}}{2}\right)}\left(\int_{0}^{t} e^{\alpha_{1} v+\beta_{1} \frac{v^{2}}{2}} d v \int_{0}^{t}\left(\alpha_{1}+\beta_{1} v\right)\right. \\
& e^{\left(\alpha_{2}-\alpha_{1}\right) v+\left(\beta_{2}-\beta_{1}\right) \frac{v^{2}}{2}} d v-\int_{0}^{t} e^{\alpha_{1} v+\beta_{1} \frac{v^{2}}{2}} \\
&\left.\left(\int_{0}^{t}\left(\alpha_{1}+\beta_{1} v\right) e^{\left(\alpha_{2}-\alpha_{1}\right) v+\left(\beta_{2}-\beta_{1}\right) \frac{v^{2}}{2}} d v\right) d v-\frac{1}{\alpha_{2}}\right)
\end{aligned}
$$

The variance of the number of customers in the entire system is

$$
\begin{aligned}
& V(t)= \lambda e^{-\left(\alpha_{1} t+\beta_{1} \frac{t^{2}}{2}\right)}\left(\int_{0}^{t} e^{\alpha_{1} v+\beta_{1} \frac{v^{2}}{2}} d v-\frac{1}{\alpha_{1}}\right) \\
& \lambda \theta e^{-\left(\alpha_{2} t+\beta_{1} \frac{t^{2}}{2}\right)}\left(\frac{1}{\alpha_{2}-\alpha_{1}}\right. \\
&\left.-\frac{\int_{0}^{t}\left(\alpha_{1}+\beta_{1} v\right) e^{\left(\alpha_{2}-\alpha_{1}\right) v+\left(\beta_{2}-\beta_{1}\right) \frac{v^{2}}{2}} d v}{\alpha_{1}}\right) \\
&+\lambda \theta e^{-\left(\alpha_{2} t+\beta_{1} \frac{t^{2}}{2}\right)}\left(\int_{0}^{t} e^{\alpha_{1} v+\beta_{1} \frac{v^{2}}{2}} d v \int_{0}^{t}\left(\alpha_{1}+\beta_{1} v\right)\right. \\
& e^{\left(\alpha_{2}-\alpha_{1}\right) v+\left(\beta_{2}-\beta_{1}\right) \frac{v^{2}}{2}} d v-\int_{0}^{t} e^{\alpha_{1} v+\beta_{1} \frac{v^{2}}{2}} \\
&\left.\left(\int_{0}^{t}\left(\alpha_{1}+\beta_{1} v\right) e^{\left(\alpha_{2}-\alpha_{1}\right) v+\left(\beta_{2}-\beta_{1}\right) \frac{v^{2}}{2}} d v\right) d v-\frac{1}{\alpha_{2}}\right)
\end{aligned}
$$

\section{NUMERICAL ILLUSTRATION AND SENSITIVITY ANALYSIS}

In this section, the performance of the proposed queueing model is discussed through numerical illustration. Different values of the parameters are considered for service rates and arrivals of customers. The transient behavior of the model is studied by computing the performance measures with the following sets of values for the model parameters: 
$\mathrm{t}=0.14,0.16,0.18,0.2 ; \quad \lambda=3,4,5,6,7 ; \quad \alpha_{1=8}, 8.4,8.8$, 9.2, 9.6; $\beta 1=5,10,15,20,25 ; \quad \alpha_{2}=10,10.4,10.8,11.2,11.6$; and $\beta_{2}=10,15,20,25,30$

The probability of emptiness of the queue, the mean number of customers, the utilization of service station, the throughput of the service station, the variance of the number of customers in the system, and the coefficient of variation of the number of customers in the system are computed, for different values of the parameters $t, \lambda, \alpha_{1}, \beta_{1}$, $\alpha_{2}, \beta_{2}$, and $\theta$ are presented in Table 1

From Table 1, it is observed that as time (t) varies from 0.14 to 0.20 , the probability of emptiness of the queue

Table 1

Values of $P_{00}(t), P_{0 .}(t), P_{.0}(t), L_{1}(t), L_{2}(t)$ for different values of parameters

\begin{tabular}{|c|c|c|c|c|c|c|c|c|c|c|c|c|c|}
\hline $\mathrm{t}$ & $\lambda$ & $\alpha_{1}$ & $\beta_{1}$ & $\alpha_{2}$ & $\beta_{2}$ & $\theta$ & $\pi$ & $P_{00}(t)$ & $P_{0 .}(t)$ & $P_{.0}(t)$ & $\mathrm{L}_{1}(\mathrm{t})$ & $\mathrm{L}_{2}(\mathrm{t})$ & $\mathrm{L}(\mathrm{t})$ \\
\hline 0.14 & 3 & 8 & 5 & 10 & 10 & 0.1 & 0.9 & 0.86547 & 0.87881 & 0.98483 & 0.12919 & 0.02871 & 0.1579 \\
\hline 0.16 & & & & & & & & 0.84033 & 0.84918 & 0.98957 & 0.16348 & 0.02114 & 0.18462 \\
\hline 0.18 & & & & & & & & 0.81969 & 0.82535 & 0.99314 & 0.19194 & 0.01532 & 0.20726 \\
\hline 0.20 & & & & & & & & 0.8028 & 0.80621 & 0.99577 & 0.21541 & 0.01089 & 0.2263 \\
\hline & 4 & & & & & & & 0.74612 & 0.75035 & 0.99437 & 0.28722 & 0.01451 & 0.30173 \\
\hline & 5 & & & & & & & 0.69345 & 0.69836 & 0.99296 & 0.35902 & 0.01814 & 0.37716 \\
\hline & 6 & & & & & & & 0.64449 & 0.64997 & 0.99156 & 0.43082 & 0.02177 & 0.45259 \\
\hline & 7 & & & & & & & 0.59894 & 0.60494 & 0.99016 & 0.50263 & 0.0254 & 0.52803 \\
\hline & & 8.4 & & & & & & 0.59236 & 0.60466 & 0.97966 & 0.50309 & 0.03606 & 0.53915 \\
\hline & & 8.8 & & & & & & 0.58312 & 0.60547 & 0.96308 & 0.50175 & 0.05313 & 0.55488 \\
\hline & & 9.2 & & & & & & 0.56565 & 0.60714 & 0.93166 & 0.49899 & 0.0863 & 0.58529 \\
\hline & & 9.6 & & & & & & 0.51498 & 0.60951 & 0.8449 & 0.4951 & 0.18405 & 0.67915 \\
\hline & & & 10 & & & & & 0.5251 & 0.62134 & 0.8451 & 0.47587 & 0.1831 & 0.65897 \\
\hline & & & 15 & & & & & 0.53486 & 0.63277 & 0.84527 & 0.45764 & 0.18361 & 0.64125 \\
\hline & & & 20 & & & & & 0.54428 & 0.64381 & 0.8454 & 0.44035 & 0.18346 & 0.62381 \\
\hline & & & 25 & & & & & 0.55335 & 0.65446 & 0.8455 & 0.42394 & 0.18334 & 0.60728 \\
\hline & & & & 10.4 & & & & 0.61328 & 0.65446 & 0.93708 & 0.42394 & 0.07876 & 0.5027 \\
\hline & & & & 10.8 & & & & 0.63386 & 0.65446 & 0.96852 & 0.42394 & 0.04423 & 0.46817 \\
\hline & & & & 11.2 & & & & 0.64386 & 0.65446 & 0.98379 & 0.42394 & 0.02723 & 0.45117 \\
\hline & & & & 11.6 & & & & 0.64954 & 0.65446 & 0.99248 & 0.42394 & 0.01726 & 0.4412 \\
\hline & & & & & 15 & & & 0.65037 & 0.65446 & 0.99374 & 0.42394 & 0.01507 & 0.43901 \\
\hline & & & & & 20 & & & 0.6511 & 0.65446 & 0.99486 & 0.42394 & 0.01311 & 0.43705 \\
\hline & & & & & 25 & & & 0.65175 & 0.65446 & 0.99585 & 0.42394 & 0.01135 & 0.43529 \\
\hline & & & & & 30 & & & 0.65233 & 0.65446 & 0.99674 & 0.42394 & 0.00978 & 0.43372 \\
\hline & & & & & & 0.2 & 0.8 & 0.6502 & 0.65446 & 0.99349 & 0.42394 & 0.01955 & 0.44349 \\
\hline & & & & & & 0.3 & 0.7 & 0.64808 & 0.65446 & 0.99024 & 0.42394 & 0.02933 & 0.45327 \\
\hline & & & & & & 0.4 & 0.6 & 0.64597 & 0.65446 & 0.98701 & 0.42394 & 0.03911 & 0.46305 \\
\hline & & & & & & 0.5 & 0.5 & 0.64386 & 0.65446 & 0.98379 & 0.42394 & 0.04888 & 0.47282 \\
\hline
\end{tabular}

decreases from 0.86547 to 0.8028 . The probability that the emptiness of the first queue decreases from 0.87881 to 0.80621 and the probability that the emptiness of the second queue increases from 0.98483 to 0.99577 . The mean number of customers in first queue increases from 0.12919 to 0.21541 , and in second queue decreases from 0.02871 to 0.01089 , when all other parameters are fixed. The probability of emptiness of the system is highly sensitive with respect to time.

It is further observed that as the arrival rate $(\lambda)$ changes from 4 to 7 , the probability of emptiness of the system, first and second queues decrease from 0.74612 to $0.59899,0.75035$ to 0.60494 and 0.99437 to 0.99016 respectively, the mean number of customers in system, first and second queue increase from 0.28722 to $0.50263,0.01451$ to 0.0254 , and 0.30173 to 0.52803 respectively, when all other parameters are fixed.

It is observed that as the service rate parameter $\left(\alpha_{1}\right)$ varies from 8.4 to 9.6, the probability of emptiness of the system and second queue decrease from 0.59236 to 0.51498 and
0.99016 to 0.8449 respectively, the probability that the emptiness of the first queue increases from 0.60466 to 0.60951, the mean number of customers in first queue decreases from 0.50309 to 0.4951 , and in second queue and in the system it increase from 0.03606 to 0.18405 and 0.53915 to 0.67915 respectively, when all other parameters are fixed. It is also observed that as the service rate parameter $\left(\beta_{1}\right)$ varies from 10 to 25 , the probability of emptiness of the system, first and second queues increase from 0.5251 to $0.55335,0.62134$ to 0.65446 and 0.8451 to 0.8455 respectively, the mean number of customers in the system, first and second queues decrease from 0.65897 to $0.60728,0.47587$ to 0.42394 and 0.18381 to 0.18334 respectively, when all other parameters are fixed.

It is observed that as the service rate parameter $\left(\alpha_{2}\right)$ increases from 10.4 to 11.6 , the probability of emptiness of the system and second queue increase from 0.61328 to 0.64954 and 0.93708 to 0.99248 respectively and in the first queue it remains constant. The mean number of customers in second queue decreases from 0.07876 to 0.01726 , but in the first queue it remains constant, when all other parameters are 
fixed. It is also observed that as the service rate parameter $\left(\beta_{2}\right)$ increases from 15 to 30 , the probability of emptiness of the first queue increase from 0.65037to 0.65233; the mean number of customers in second queue decreases from 0.01507 to 0.00978 , but in the first queue it remains constant, when all other parameters are fixed.
It is also observed that as the service rate parameter $(\theta)$ increases from 0.2 to 0.5 , the probability of emptiness of in the second queue decreases from 0.99349 to 0.98379 . The mean number of customers in second queue increases from 0.01955 to 0.04888 , but in the first queue it remains constant, when all other parameters are fixed.

Table 2

Values of $U_{1}(t), U_{2}(t), T_{1}(t), \operatorname{Thp}_{2}(t), W_{1}(t), W_{2}(t)$ for different values of parameters

\begin{tabular}{|c|c|c|c|c|c|c|c|c|c|c|c|c|c|}
\hline t & $\lambda$ & $\alpha_{1}$ & $\beta_{1}$ & $\alpha_{2}$ & $\beta_{2}$ & $\theta$ & $\pi$ & $\mathrm{U}_{1}(\mathrm{t})$ & $\mathrm{U}_{2}(\mathrm{t})$ & $\operatorname{Thp}_{1}(\mathrm{t})$ & $\operatorname{Thp}_{2}(\mathrm{t})$ & $\mathrm{W}_{1}(\mathrm{t})$ & $\mathrm{W}_{2}(\mathrm{t})$ \\
\hline 0.14 & 3 & 8 & 5 & 10 & 10 & 0.1 & 0.9 & 0.12119 & 0.01517 & 1.05437 & 0.17299 & 0.12253 & 0.08839 \\
\hline 0.16 & & & & & & & & 0.15082 & 0.01043 & 1.3272 & 0.12098 & 0.12318 & 0.08666 \\
\hline 0.18 & & & & & & & & 0.17465 & 0.00686 & 1.55435 & 0.08099 & 0.12349 & 0.08504 \\
\hline 0.20 & & & & & & & & 0.19379 & 0.00423 & 1.74412 & 0.05075 & 0.12351 & 0.08351 \\
\hline & 4 & & & & & & & 0.24965 & 0.00563 & 2.24685 & 0.06762 & 0.12783 & 0.08357 \\
\hline & 5 & & & & & & & 0.30164 & 0.00704 & 2.71476 & 0.08446 & 0.13225 & 0.08363 \\
\hline & 6 & & & & & & & 0.35003 & 0.00844 & 3.15024 & 0.10128 & 0.13676 & 0.08369 \\
\hline & 7 & & & & & & & 0.39506 & 0.00984 & 3.55555 & 0.11808 & 0.14136 & 0.08375 \\
\hline & & 8.4 & & & & & & 0.39534 & 0.02034 & 3.71618 & 0.2441 & 0.13538 & 0.08419 \\
\hline & & 8.8 & & & & & & 0.39453 & & 3.86642 & & & \\
\hline & & 9.2 & & & & & & 0.39286 & 0.06864 & 4.00716 & 0.82005 & 0.12453 & 0.08632 \\
\hline & & 9.6 & & & & & & 0.39049 & 0.1551 & 4.13916 & 1.86119 & 0.11961 & 0.09055 \\
\hline & & & 10 & & & & & 0.37866 & 0.1549 & 4.39244 & 1.85874 & 0.10834 & 0.09054 \\
\hline & & & 15 & & & & & 0.36723 & 0.15473 & 4.62707 & 1.85677 & 0.09891 & 0.09053 \\
\hline & & & 20 & & & & & 0.35619 & 0.1546 & 4.84417 & 1.85522 & 0.0909 & 0.09053 \\
\hline & & & 25 & & & & & 0.34554 & 0.15456 & 5.04482 & 1.85405 & 0.08403 & 0.09052 \\
\hline & & & & 10.4 & & & & 0.34554 & 0.06292 & 5.04482 & 0.78022 & 0.08403 & 0.08329 \\
\hline & & & & 10.8 & & & & 0.34554 & 0.03148 & 5.04482 & 0.40297 & 0.08403 & 0.07938 \\
\hline & & & & 11.2 & & & & 0.34554 & 0.01621 & 5.04482 & 0.21392 & 0.08403 & 0.07638 \\
\hline & & & & 11.6 & & & & 0.34554 & 0.00752 & 5.04482 & 0.10231 & 0.08403 & 0.07381 \\
\hline & & & & & 15 & & & 0.34554 & 0.00626 & 5.04482 & 0.09145 & 0.08403 & 0.06871 \\
\hline & & & & & 20 & & & 0.34554 & 0.00514 & 5.04482 & 0.08022 & 0.08403 & 0.06427 \\
\hline & & & & & 25 & & & 0.34554 & 0.00415 & 5.04482 & 0.06883 & 0.08403 & 0.06037 \\
\hline & & & & & 30 & & & 0.34554 & 0.00326 & 5.04482 & 0.05742 & 0.08403 & 0.05691 \\
\hline & & & & & & 0.2 & 0.8 & 0.34554 & 0.00651 & 5.04482 & 0.11465 & 0.08403 & 0.057 \\
\hline & & & & & & 0.3 & 0.7 & 0.34554 & 0.00976 & 5.04482 & 0.17169 & 0.08403 & 0.0571 \\
\hline & & & & & & 0.4 & 0.6 & 0.34554 & 0.01299 & 5.04482 & 0.22854 & 0.08403 & 0.05719 \\
\hline & & & & & & 0.5 & 0.5 & 0.34554 & 0.01621 & 5.04482 & 0.28522 & 0.08403 & 0.05728 \\
\hline
\end{tabular}

From Table 2, as time (t) varies from 0.14 to 0.20 , the utilization of first service station, the throughput of first service station, and the average waiting time of a customers in first queue increase from 0.12119 to0.19379, 1.05437 to 1.74412 and 0.12253 to 0.12351 respectively. But in second queue they decrease from 0.01517 to $0.00423,0.17299$ to 0.05075 and 0.08839 to 0.08351 respectively, when all other parameters are fixed. It is observed that the utilization of the service stations, throughput of the service stations, and the waiting time of a customer in each queue are highly sensitive with respect to time.

It is further observed that as the arrival parameter $(\lambda)$ varies from 4 to 7 , the utilization of service station, the throughput of service station, the average waiting time of a customers in first and second queues increase from 0.24965 to 0.39506 , 0.00563 to $0.00984,2.24685$ to $3.5555,0.06762$ to 0.11808 , 0.12783 to $0.14136,0.08357$ to 0.08375 respectively, when all other parameters are fixed. It is also observed that as the service rate parameter $\left(\alpha_{1}\right)$ varies from 8.4 to 9.6 , the utilization of first service station decreases from 0.39534 to 0.39049 , and in the second service station it increases from 0.02034 to 0.1551 . The throughput of first and second service stations increase from 3.71618 to 4.13916 and 0.2441 to 1.86119 respectively. The average waiting time of a customers in first queue decreases from 0.13538 to 0.11961 , and in second queue increases from 0.08419 to 0.09055 , when all other parameters are fixed. It is also observed that as the service rate parameter $\left(\beta_{1}\right)$ increases from 10 to 25 , the utilization of first and second service stations decrease from 0.37866 to 0.34554 and 0.1549 to 0.1545 respectively. The throughput of first service station increases from 4.39244 to 5.04482 and in second service station decreases from 1.85874 to 1.85405 . The average waiting time of a customers in first and second queues decrease from 0.10834 to 0.08403 and 0.09053 to 0.09052 , respectively, when all other parameters are fixed.

It is also observed that as the service rate parameter $\left(\alpha_{2}\right)$ and $\left(\beta_{2}\right)$ varies the utilization of second service station decreases, the throughput of second of service station decreases, the average waiting time of customers in second queue decrease. With respect to the variations in $\alpha_{2}$ and $\beta_{2}$ the performance measures remains constant in the first queue, when all other parameters are fixed. 
It is also observed that as the service rate parameter $(\theta)$ increases, the utilization of service station in second queue increases from 0.00651 to 0.01621 . The throughput of second service station increases from 0.11465 to 0.28522 , the average waiting time of customers in second queue increases from 0.057 to 0.05728 , when all other parameters are fixed.

From Table 3, as time (t) varies from 0.14 to 0.2 ; the variance of the number of customers in first queue increases from
0.12919 to 0.21541 , and in second queue it decreases from 0.02871 to 0.01089 , and in the system it increases from 0.1579 to 0.2263 . The coefficient of variation of number of customers in first queue decreases from 278.21997 to 215.45922, and in second queue it increases from 808.68511 to 1536.11 when all other parameters are fixed.

Table 3

Values of $V_{1}(t), V_{2}(t), C v_{1}(t), C_{2}(t)$ for different values of parameters

\begin{tabular}{|c|c|c|c|c|c|c|c|c|c|c|c|c|}
\hline $\mathrm{t}$ & $\lambda$ & $\alpha_{1}$ & $\beta_{1}$ & $\alpha_{2}$ & $\beta_{2}$ & $\theta$ & $\pi$ & $\mathrm{V} 1(\mathrm{t})$ & $\mathrm{V} 2(\mathrm{t})$ & $\mathrm{V}(\mathrm{t})$ & $\mathrm{Cv} 1(\mathrm{t})$ & $\mathrm{Cv}(\mathrm{t})$ \\
\hline 0.14 & 3 & 8 & 5 & 10 & 10 & 0.1 & 0.9 & 0.12919 & 0.02871 & 0.1579 & 278.21997 & 808.68511 \\
\hline 0.16 & & & & & & & & 0.16348 & 0.02114 & 0.18462 & 247.32313 & 976.63225 \\
\hline 0.18 & & & & & & & & 0.19194 & 0.01532 & 0.20726 & 228.25171 & 1204.97 \\
\hline 0.20 & & & & & & & & 0.21541 & 0.01089 & 0.2263 & 215.45922 & 1536.11 \\
\hline & 4 & & & & & & & 0.28722 & 0.01451 & 0.30173 & 186.59316 & 1330.31 \\
\hline & 5 & & & & & & & 0.35902 & 0.01814 & 0.37716 & 166.894 & 1189.87 \\
\hline & 6 & & & & & & & 0.43082 & 0.02177 & 0.45259 & 152.35268 & 1086.19 \\
\hline & 7 & & & & & & & 0.50263 & 0.0254 & 0.52803 & 141.05117 & 1005.62 \\
\hline & & 8.4 & & & & & & 0.50309 & 0.03606 & 0.53915 & 140.98691 & 697.56173 \\
\hline & & 8.8 & & & & & & 0.50175 & 0.05313 & 0.55488 & 141.17389 & 515.59868 \\
\hline & & 9.2 & & & & & & 0.49899 & 0.0863 & 0.58529 & 141.5639 & 375.86408 \\
\hline & & 9.6 & & & & & & 0.4951 & 0.18405 & 0.67915 & 142.12016 & 243.58641 \\
\hline & & & 10 & & & & & 0.47587 & 0.1831 & 0.65897 & 144.96189 & 243.76125 \\
\hline & & & 15 & & & & & 0.45764 & 0.18361 & 0.64125 & 147.82094 & 243.90218 \\
\hline & & & 20 & & & & & 0.44035 & 0.18346 & 0.62381 & 150.69582 & 244.01304 \\
\hline & & & 25 & & & & & 0.42394 & 0.18334 & 0.60728 & 153.58502 & 244.09731 \\
\hline & & & & 10.4 & & & & 0.42394 & 0.07876 & 0.5027 & 153.58502 & 392.26925 \\
\hline & & & & 10.8 & & & & 0.42394 & 0.04423 & 0.46817 & 153.58502 & 559.11726 \\
\hline & & & & 11.2 & & & & 0.42394 & 0.02723 & 0.45117 & 153.58502 & 782.33369 \\
\hline & & & & 11.6 & & & & 0.42394 & 0.01726 & 0.4412 & 153.58502 & 1150.77 \\
\hline & & & & 15 & & & 0.42394 & 0.01507 & 0.43901 & 153.58502 & 1261.58 \\
\hline & & & & & 20 & & & 0.42394 & 0.01311 & 0.43705 & 153.58502 & 1392.68 \\
\hline & & & & 25 & & & 0.42394 & 0.01135 & 0.43529 & 153.58502 & 1551.36 \\
\hline & & & & 30 & & & 0.42394 & 0.00978 & 0.43372 & 153.58502 & 1749.37 \\
\hline & & & & & & 0.2 & 0.8 & 0.42394 & 0.01955 & 0.44349 & 153.58502 & 1236.99 \\
\hline & & & & & & 0.3 & 0.7 & 0.42394 & 0.02933 & 0.45327 & 153.58502 & 1010 \\
\hline & & & & & & 0.4 & 0.6 & 0.42394 & 0.03911 & 0.46305 & 153.58502 & 874.68747 \\
\hline & & & & & & 0.5 & 0.5 & 0.42394 & 0.04888 & 0.47282 & 153.58502 & 782.34426 \\
\hline
\end{tabular}

It is further observed that as the arrival parameter $(\lambda)$ varies from 4 to 7 , the variance of the number of customers in each queue and in the system increase from 0.28722 to 0.50263 , 0.01451 to 0.0254 and 0.30173 to 0.52803 respectively. The coefficient of variation of number of customers in first and second queues decreases from 186.59316 to 141.05117 and 1330.31 to 1005.62 respectively, when all other parameters are fixed. It is also observed that as the service rate $\left(\alpha_{1}\right)$ varies from 8.4 to 9.6 , the variance of the number of customers in first queue decrease from 0.50309 to 0.4951 , and in second queue it increases from 0.03606 to 0.18405 . The coefficient of variation of number of customers in first queue increases from 140.98691 to 142.12016 , and in second queue decreases from 697.56173 to 243.58641 , when all other parameters are fixed. It is also observed that as the service rate parameter $\left(\beta_{1}\right)$ varies from 10 to 25 , the variance of the number of customers in first and second queues from 0.47587 to 0.42394 and 0.18381 to 0.18334 respectively; the coefficient of variation of number of customers in each queue increases from 144.96189 to 153.58502 , when all other parameters are fixed.

It is also observed that as the service rate parameters $\left(\alpha_{2}\right)$ and $\left(\beta_{2}\right)$ varies from 10.4 to 11.6 and 15 to 30 respectively, the variance of the number of customers in second queue and entire queue decreases. The coefficient of variation of number of customers in second queue decreases, when all other parameters are fixed.

It is also observed that as the service rate $(\theta)$ increases from 0.2 to 0.5 , the variance of the number of customers in second queue increases from 0.01955 to 0.4888 the coefficient of variation of number of customers in second queue decreases from 1236.99 to 782.34426 , when all other parameters are fixed.

\section{SENSITIVITY ANALYSIS}

Sensitivity analysis of the model is performed with respect to the value of time $(\mathrm{t})$, arrival rate $\lambda$, service rates of the first, second servers $\mu_{1}(t)$ and $\mu_{2}(t)$, and all parameters together on the mean number of customers in the first, second queues ,the utilization of service station in first, second queues ,the mean delay in the first, second queues and the throughput of service station in first and second queues. 
For different values of $t, \lambda, \alpha_{1}, \beta_{1}, \alpha_{2}, \beta_{2}$ the mean number of customers in the first and second queue, the utilization of service station in first and second queue, the mean delay in the first queue and second queue and the throughput of service station in first and second queue are computed with variation of $-10 \%,-5 \%, 0 \%, 5 \%, 10 \%$ are computed and are given in Table 4 .
From Table 4 it is observed that the performance measures are highly affected by the variations in the time and other parameters of the model.

Table 4

The values of $L_{1}(t), L_{2}(t), U_{1}(t), U_{2}(t), T h p_{1}(t), T h p_{2}(t), W_{1}(t), W_{2}(t)$ for different values of $t, \lambda, \alpha_{1}, \beta_{1}, \alpha_{2}$, and $\beta_{2}$.

\begin{tabular}{|c|c|c|c|c|c|c|}
\hline \multirow[b]{2}{*}{ Parameter } & \multirow{2}{*}{$\begin{array}{l}\text { Performance } \\
\text { measure }\end{array}$} & \multicolumn{5}{|c|}{$\%$ change in parameters } \\
\hline & & -10 & -5 & 0 & 5 & 10 \\
\hline \multirow[t]{8}{*}{$\mathrm{t}=0.20$} & L1(t) & 0.45587 & 0.49853 & 0.50053 & 0.50252 & 0.5362 \\
\hline & $\mathrm{L} 2(\mathrm{t})$ & 0.04837 & 0.03605 & 0.03549 & 0.03493 & 0.02572 \\
\hline & $\mathrm{U} 1(\mathrm{t})$ & 0.3661 & 0.39258 & 0.39379 & 0.39499 & 0.41503 \\
\hline & $\mathrm{U} 2(\mathrm{t})$ & 0.03076 & 0.02227 & 0.02188 & 0.0215 & 0.01521 \\
\hline & $\mathrm{W} 1(\mathrm{t})$ & 0.12578 & 0.12705 & 0.12711 & 0.12716 & 0.12792 \\
\hline & $\mathrm{W} 2(\mathrm{t})$ & 0.08258 & 0.08097 & 0.08089 & 0.08081 & 0.07935 \\
\hline & Thp1(t) & 3.6244 & 3.92379 & 3.93793 & 3.95191 & 4.19182 \\
\hline & Thp2(t) & 0.37836 & 0.27812 & 0.2735 & 0.26895 & 0.19321 \\
\hline \multirow[t]{8}{*}{$\lambda=7$} & L1(t) & 0.45048 & 0.47551 & 0.50053 & 0.52556 & 0.55059 \\
\hline & $\mathrm{L} 2(\mathrm{t})$ & 0.03194 & 0.03372 & 0.03549 & 0.03727 & 0.03904 \\
\hline & $\mathrm{U} 1(\mathrm{t})$ & 0.36268 & 0.37843 & 0.39379 & 0.40878 & 0.42339 \\
\hline & $\mathrm{U} 2(\mathrm{t})$ & 0.01971 & 0.0208 & 0.02188 & 0.02296 & 0.02404 \\
\hline & $\mathrm{W} 1(\mathrm{t})$ & 0.12421 & 0.12565 & 0.12711 & 0.12857 & 0.13004 \\
\hline & W2(t) & 0.0808 & 0.08084 & 0.08089 & 0.08093 & 0.08098 \\
\hline & Thp1(t) & 3.62678 & 3.7843 & 3.93793 & 4.08776 & 4.23388 \\
\hline & Thp2(t) & 0.24642 & 0.25997 & 0.2735 & 0.28702 & 0.30052 \\
\hline \multirow[t]{8}{*}{$\alpha 1=9$} & L1(t) & 0.50293 & 0.50278 & 0.50053 & 0.49667 & 0.49157 \\
\hline & L2(t) & 0.01595 & 0.02373 & 0.03549 & 0.05644 & 0.1074 \\
\hline & $\mathrm{U} 1(\mathrm{t})$ & 0.39525 & 0.39515 & 0.39379 & 0.39145 & 0.38833 \\
\hline & $\mathrm{U} 2(\mathrm{t})$ & 0.00257 & 0.01031 & 0.02188 & 0.04216 & 0.08975 \\
\hline & $\mathrm{W} 1(\mathrm{t})$ & 0.13983 & 0.13323 & 0.12711 & 0.12142 & 0.11613 \\
\hline & $\mathrm{W} 2(\mathrm{t})$ & 0.0801 & 0.08042 & 0.08089 & 0.08174 & 0.08382 \\
\hline & Thp1(t) & 3.59674 & 3.77369 & 3.93793 & 4.09064 & 4.23284 \\
\hline & Thp2(t) & 0.03222 & 0.12888 & 0.2735 & 0.52695 & 1.12181 \\
\hline \multirow[t]{8}{*}{$\beta 1=5$} & L1(t) & 0.5025 & 0.50151 & 0.50053 & 0.49955 & 0.49858 \\
\hline & L2(t) & 0.03552 & 0.03551 & 0.03549 & 0.03547 & 0.03546 \\
\hline & $\mathrm{U} 1(\mathrm{t})$ & 0.39498 & 0.39439 & 0.39379 & 0.3932 & 0.39261 \\
\hline & $\mathrm{U} 2(\mathrm{t})$ & 0.02191 & 0.0219 & 0.02188 & 0.02186 & 0.02185 \\
\hline & W1(t) & 0.12851 & 0.1278 & 0.12711 & 0.12642 & 0.12573 \\
\hline & $\mathrm{W} 2(\mathrm{t})$ & 0.08089 & 0.08089 & 0.08089 & 0.08089 & 0.08089 \\
\hline & Thp1(t) & 3.91033 & 3.92415 & 3.93793 & 3.95165 & 3.96532 \\
\hline & Thp2(t) & 0.27391 & 0.2737 & 0.2735 & 0.2733 & 0.2731 \\
\hline \multirow[t]{8}{*}{$\alpha 2=10.5$} & L1(t) & 0.50053 & 0.50053 & 0.50053 & 0.50053 & 0.50053 \\
\hline & $\mathrm{L} 2(\mathrm{t})$ & 0.18173 & 0.06889 & 0.03549 & 0.01979 & 0.01087 \\
\hline & $\mathrm{U} 1(\mathrm{t})$ & 0.39379 & 0.39379 & 0.39379 & 0.39379 & 0.39379 \\
\hline & $\mathrm{U} 2(\mathrm{t})$ & 0.15076 & 0.05187 & 0.02188 & 0.00829 & 0.00102 \\
\hline & W1(t) & 0.12711 & 0.12711 & 0.12711 & 0.12711 & 0.12711 \\
\hline & W2(t) & 0.09467 & 0.08575 & 0.08089 & 0.0771 & 0.07384 \\
\hline & Thp1(t) & 3.93793 & 3.93793 & 3.93793 & 3.93793 & 3.93793 \\
\hline & Thp2(t) & 1.72616 & 0.62111 & 0.2735 & 0.10801 & 0.01383 \\
\hline \multirow[t]{6}{*}{$\beta 2=10$} & L1(t) & 0.50053 & 0.50053 & 0.50053 & 0.50053 & 0.50053 \\
\hline & $\mathrm{L} 2(\mathrm{t})$ & 0.03635 & 0.03592 & 0.03549 & 0.03507 & 0.03465 \\
\hline & $\mathrm{U1}(\mathrm{t})$ & 0.39379 & 0.39379 & 0.39379 & 0.39379 & 0.39379 \\
\hline & $\mathrm{U} 2(\mathrm{t})$ & 0.02245 & 0.02216 & 0.02188 & 0.0216 & 0.02132 \\
\hline & $\mathrm{W} 1(\mathrm{t})$ & 0.12711 & 0.12711 & 0.12711 & 0.12711 & 0.12711 \\
\hline & $\mathrm{W} 2(\mathrm{t})$ & 0.08223 & 0.08155 & 0.08089 & 0.08023 & 0.07959 \\
\hline
\end{tabular}




\begin{tabular}{|l|l|l|l|l|l|l|}
\hline \multirow{5}{*}{$\theta=0.1$} & Thp1(t) & 3.93793 & 3.93793 & 3.93793 & 3.93793 & 3.93793 \\
\cline { 2 - 7 } & Thp2(t) & 0.27615 & 0.27484 & 0.2735 & 0.27215 & 0.27077 \\
\hline & L1(t) & 0.50053 & 0.50053 & 0.50053 & 0.50053 & 0.50053 \\
\cline { 2 - 7 } & L2(t) & 0.03194 & 0.03372 & 0.03549 & 0.03727 & 0.03904 \\
\cline { 2 - 7 } & U1(t) & 0.39379 & 0.39379 & 0.39379 & 0.39379 & 0.39379 \\
\cline { 2 - 7 } & U2(t) & 0.01971 & 0.0208 & 0.02188 & 0.02296 & 0.02404 \\
\cline { 2 - 7 } & W1(t) & 0.12711 & 0.12711 & 0.12711 & 0.12711 & 0.12711 \\
\cline { 2 - 7 } & W2(t) & 0.0808 & 0.08084 & 0.08089 & 0.08093 & 0.08098 \\
\cline { 2 - 7 } & Thp1(t) & 3.93793 & 3.93793 & 3.93793 & 3.93793 & 3.93793 \\
\cline { 2 - 7 } & Thp2(t) & 0.24642 & 0.25997 & 0.2735 & 0.28702 & 0.30052 \\
\hline
\end{tabular}

\section{COMPARATIVE STUDY:}

The comparative study of the developed models with homogenous Poisson arrivals is presented in this section. The performance measure of both models are presented in the Table 5 for different values of $\mathrm{t}=0.14,0.16,0.18,2$.

Table 5

Comparative study of models with Non-Homogeneous and Homogeneous Poisson service rates

\begin{tabular}{|c|c|c|c|c|c|}
\hline $\mathrm{t}$ & $\begin{array}{l}\text { Param } \\
\text { eter } \\
\text { Measu } \\
\text { re }\end{array}$ & $\begin{array}{c}\text { Non- } \\
\text { Homog } \\
\text { eneous } \\
\text { service } \\
\text { rate } \\
\end{array}$ & $\begin{array}{l}\text { Homoge } \\
\text { neous } \\
\text { service } \\
\text { rate }\end{array}$ & $\begin{array}{c}\text { Differenc } \\
\mathrm{e}\end{array}$ & $\begin{array}{l}\text { Percenta } \\
\text { ge of } \\
\text { Variatio } \\
n\end{array}$ \\
\hline \multirow[t]{6}{*}{0.14} & $\mathrm{~L}_{1}(\mathrm{t})$ & 0.12919 & 0.13029 & 0.0011 & 0.85146 \\
\hline & $\mathrm{L}_{2}(\mathrm{t})$ & 0.02871 & 0.03244 & 0.00373 & 12.992 \\
\hline & $\mathrm{U}_{1}(\mathrm{t})$ & 0.12119 & 0.12216 & 0.00097 & 0.8004 \\
\hline & $\mathrm{U}_{2}(\mathrm{t})$ & 0.01517 & 0.01748 & 0.00231 & 15.2274 \\
\hline & $\mathrm{W}_{1}(\mathrm{t})$ & 0.12253 & 0.13332 & 0.01079 & 8.80601 \\
\hline & $\mathrm{W}_{2}(\mathrm{t})$ & 0.08839 & 0.10088 & 0.01249 & 14.1306 \\
\hline \multirow[t]{6}{*}{0.16} & $\mathrm{~L}_{1}(\mathrm{t})$ & 0.16348 & 0.16647 & 0.00299 & 1.82897 \\
\hline & $\mathrm{L}_{2}(\mathrm{t})$ & 0.02114 & 0.02492 & 0.00378 & 17.8808 \\
\hline & $\mathrm{U}_{1}(\mathrm{t})$ & 0.15082 & 0.15335 & 0.00253 & 1.6775 \\
\hline & $\mathrm{U}_{2}(\mathrm{t})$ & 0.01043 & 0.01272 & 0.00229 & 21.9559 \\
\hline & $\mathrm{W}_{1}(\mathrm{t})$ & 0.12318 & 0.13569 & 0.01251 & 10.1559 \\
\hline & $\mathrm{W}_{2}(\mathrm{t})$ & 0.08666 & 0.10064 & 0.01398 & 16.132 \\
\hline \multirow[t]{6}{*}{0.18} & $\mathrm{~L}_{1}(\mathrm{t})$ & 0.19194 & 0.1973 & 0.00536 & 2.79254 \\
\hline & $\mathrm{L}_{2}(\mathrm{t})$ & 0.01532 & 0.01901 & 0.00369 & 24.0862 \\
\hline & $\mathrm{U}_{1}(\mathrm{t})$ & 0.17465 & 0.17906 & 0.00441 & 2.52505 \\
\hline & $\mathrm{U}_{2}(\mathrm{t})$ & 0.00686 & 0.00905 & 0.00219 & 31.9242 \\
\hline & $\mathrm{W}_{1}(\mathrm{t})$ & 0.12349 & 0.13774 & 0.01425 & 11.5394 \\
\hline & $\mathrm{W}_{2}(\mathrm{t})$ & 0.08504 & 0.10046 & 0.01542 & 18.1326 \\
\hline \multirow[t]{6}{*}{0.2} & $\mathrm{~L}_{1}(\mathrm{t})$ & 0.21541 & 0.22358 & 0.00817 & 3.79277 \\
\hline & $\mathrm{L}_{2}(\mathrm{t})$ & 0.01089 & 0.01438 & 0.00349 & 32.0478 \\
\hline & $\mathrm{U}_{1}(\mathrm{t})$ & 0.19379 & 0.20035 & 0.00656 & 3.38511 \\
\hline & $\mathrm{U}_{2}(\mathrm{t})$ & 0.00423 & 0.00624 & 0.00201 & 47.5177 \\
\hline & $\mathrm{W}_{1}(\mathrm{t})$ & 0.12351 & 0.13949 & 0.01598 & 12.9382 \\
\hline & $\mathrm{W}_{2}(\mathrm{t})$ & 0.08351 & 0.10031 & 0.0168 & 20.1174 \\
\hline
\end{tabular}

From Table 5, it is observed that as time increases the percentage variation of the performance measures between the two models also increasing. It is observed that the assumption of non-homogeneous Poisson service process has a significant influence on all the performance measures of the queueing model. Also time has a significant effect on the system performance and the proposed model can predict the performance more accurately.

\section{CONCLUSION}

This paper deals with a novel and useful two node tandem queueing model in which the service rates are time and state dependent. This model in very useful in analyzing systems under transient conditions, for the communication networks, such as LAN, WAN, MAN, in which the service rate is time dependent. This phenomenon is incorporated by characterizing the service processes through nonhomogeneous Poisson processes. The phase type service allowing the intermediary leavings from the system makes the model more effective and reduces congestion in buffers. The explicit expressions for the system performance measures such as the content of the buffers, the average waiting time of the customers in the queue and in the system, the throughput of the nodes and the variance are useful for predicting the system behavior under transient condition. It is observed that the time dependent and state dependent nature of the service processes has significant off influence on system performance measures. There is a scope for further extension of this paper by developing and analyzing a two node tandem queueing model with bulk arrivals having time and state dependent service rates. In bulk arrivals we may assume that customers may arrive batches of random size and join the queue for getting service. The bulk size distribution may be Geometric / truncated Bionomial / Uniform. This requires further investigation and which will be taken up elsewhere

\section{REFERENCES}

[1] Davis, J.L., Massey W.A., and Whitty, W., (1995) “ Sensitivity to the service- time distribution in the nonstationary Erlang loss model'’. Management Science. Vol.41, No 6, PP 1107-1116.

[2] Duffield, N.G., Massey, W.A., and Whitt,W., (2001) “ A nonstationary offered load model for packet networks". Telecommunication systems. Vol.13, Issue 3/4, PP 271296.

[3] Durga aparajitha J.,Rajkumak G.V.S, (2014)“single server queueing model with time and state dependent service rate". Journal of the Indian society for probability and statistics. Vol.15, PP 67-77.

[4] Mandelbaum, A. and Massey, W.A., (1995) "Strong approximations for time dependent queues". MOR. Vol.20, No1, PP 33-64.

[5] Massey, W.A. and Whitt, W. (1993), "Networks of infinite-server queues with nonstationary Poisson input queueing systems." Queueing systems and their applications. Vol.13, No1, PP 183-250.

[6] Massey, W.A. and Whitt, W. (1994), “An analysis of the modified load approximation for the nonstationary 
Erlang loss model',. Annals of applied probability Vol.4, No 4, PP 1145-1160.

[7] Newell, G.F. (1968), "Queues with time-dependent arrival rates (parts I-III)". Journal of Applied probability. Vol.5, PP 436-451(I), 579-590 (II), 591606 II).

[8] Rothkopf, M.H. and Oren, S.S, (1979) "A closure approximation for the nonstationary $\mathrm{M} / \mathrm{M} / \mathrm{s}$ queue". Mangement Science Vol.25, PP 522:534.

[9] Sadu, A. R., Srinivasa rao, K., Nirupama devi, K, "Forked queueing model with load dependent service rate bulk arrivals". International journal of operation research, Vol.30, No1, PP 1-32.

[10] Srinivasarao, K., Padmavathi, G., and Reddy, K.V.V.S. (2009), Performance evaluation of parallel and series communication network with dynamic bandwidth allocation, CIIT International Journal of Networking and Communication Engineering, Vol. 1, No. 7, pp 410-421.

[11] Srinivasa Rao, K., M.Govinda Rao and K.Naveen Kuamr (2011) - Transient analysis of an interdependent forked Tandem queueing model with load dependent service rate, International Journal of Computer Applications (IJCA), Volume 34, No. 3, pp: 33 - 40.

[12] Srinivasa Rao,K., VAsanta, M.R., and Vijaya Kumar, C. V. R.S., (2000), on an interdependent communication Network, opsearch.37(2):134-143.

[13] Suhasini, A.V.S. Srinivasa rao, K., Reddy, P.R.S (2012), Transient Analysis of Tandem Queuing model with non-homogeneous Poisson bulk arrivals having state dependent service rates, Sri Venkateswara University, Department of Statistics, Andhra University, Vol. 3, Issue. 3, pp 272-289.

[14] Ward Whitt (2016), "Recent papers on the time-varying single-server queue".//http.pdfs.semanticsscholar.org//

[15] William A. Massey (1996), "Stability for queues with time varying rates". Stochastic Networks of the series lecture notes in statistics 117: 95-107.

[16] William A. Massey (2002), "The analysis of queues with time varying rates for telecommunication models". Telecommunication system 21:2-4,173-204. 